\title{
Large Higgs-electron Yukawa coupling in 2HDM
}

\author{
Avital Dery, Claudia Frugiuele and Yosef Nir \\ Department of Particle Physics and Astrophysics, Weizmann Institute of Science, \\ Rehovot, 7610001 Israel \\ E-mail: avital.dery@weizmann.ac.il, claudia.frugiuele@weizmann.ac.il, \\ yosef.nir@weizmann.ac.il
}

ABSTRACT: The present upper bound on $\kappa_{e}$, the ratio between the electron Yukawa coupling and its Standard Model value, is of $\mathcal{O}(600)$. We ask what would be the implications in case that $\kappa_{e}$ is close to this upper bound. The simplest extension that allows for such enhancement is that of two Higgs doublet models (2HDM) without natural flavor conservation. In this framework, we find the following consequences: (i) Under certain conditions, measuring $\kappa_{e}$ and $\kappa_{V}$ would be enough to predict values of Yukawa couplings for other fermions and for the $H$ and $A$ scalars. (ii) In the case that the scalar potential has a softly broken $Z_{2}$ symmetry, the second Higgs doublet must be light, but if there is hard breaking of the symmetry, the second Higgs doublet can be much heavier than the electroweak scale and still allow the electron Yukawa coupling to be very different from its SM value. (iii) $\mathrm{CP}$ must not be violated at a level higher than $\mathcal{O}\left(0.01 / \kappa_{e}\right)$ in both the scalar potential and the Yukawa sector. (iv) LHC searches for $e^{+} e^{-}$resonances constrain this scenario in a significant way. Finally, we study the implications for models where one of the scalar doublets couples only to the first generation, or only to the third generation.

Keywords: Beyond Standard Model, Higgs Physics

ARXIV EPRINT: 1712.04514 


\section{Contents}

1 Introduction 1

2 Yukawa couplings in 2HDM 3

$2.1 \kappa_{e}$ in the " $\beta_{e}$-basis" 3

$2.2 \kappa_{f}$ for $f \neq e \quad 4$

$2.3 \kappa_{f}^{X}$ for $X=A, H$

$\begin{array}{lll}3 & \text { The scalar spectrum } & 6\end{array}$

$\begin{array}{lll}3.1 m_{A}^{2} \gg v^{2} & 7\end{array}$

$3.2 \lambda_{6}=\lambda_{7}=0 \quad 8$

$\begin{array}{lll}4 & \text { CP violation } & 9\end{array}$

4.1 The scalar sector $\quad 9$

$\begin{array}{ll}4.2 & \text { The Yukawa sector } \\ \end{array}$

5 LHC phenomenology 11

$5.1 A^{0}, H^{0}$ and $H^{ \pm}$decay modes 11

$\begin{array}{lll}5.2 & \text { Multi-electron signatures } & 12\end{array}$

$\begin{array}{lll}5.3 & \text { Large first generation Yukawa couplings } & 13\end{array}$

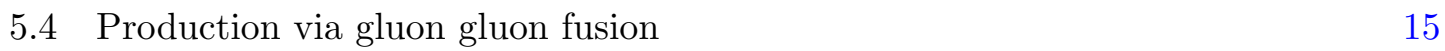

$\begin{array}{lll}5.5 & \text { Future prospects } & 15\end{array}$

$6 \quad 2 H D M$ in the literature $\quad 16$

$\begin{array}{ll}\text { 6.1 Separating the third generation from the first two } & 16\end{array}$

$\begin{array}{ll}\text { 6.2 Separating the muon from the other fermions } & 16\end{array}$

$\begin{array}{lll}7 & \text { Conclusions } & 17\end{array}$

\section{Introduction}

The Standard Model (SM) predicts the values of the Yukawa couplings. The diagonal couplings are proportional to the corresponding fermion masses,

$$
y_{f} \equiv Y_{f f}^{\mathrm{SM}}=\sqrt{2} m_{f} / v,
$$

while off-diagonal couplings vanish. As concerns the diagonal couplings, the LHC experiments are testing the SM predictions $\kappa_{f}=1$ and $\tilde{\kappa}_{f}=0$, where

$$
\begin{aligned}
\kappa_{f} & \equiv \mathcal{R} e\left(Y_{f f} / Y_{f f}^{\mathrm{SM}}\right), \\
\tilde{\kappa}_{f} & \equiv \mathcal{I} m\left(Y_{f f} / Y_{f f}^{\mathrm{SM}}\right) .
\end{aligned}
$$


While measurements of the third generation Yukawa couplings imply that $\kappa_{t, b, \tau}=\mathcal{O}(1)$ [1-4], direct measurements still allow the Yukawa couplings of the first two generations to be very different from the SM values. For the second generation, there is a mild upper bound on the charm Yukawa [5], $\kappa_{c} \lesssim 10$, and a significant constraint on the muon Yukawa [6], $\kappa_{\mu} \lesssim 1.7$. The first generation Yukawa couplings can still be orders of magnitude larger than their SM values. Moreover, there is theoretical motivation to consider a different source for the Yukawa couplings of the first two (or just the first) generations that would explain their smallness (see. e.g., [7-9]). We ask here whether indeed the Yukawa couplings of light fermions could be very different from their SM values, i.e. $\kappa \gg 1$ or $\kappa \ll 1$. For concreteness, we study the lightest charged fermion, $\kappa_{e}$.

As concerns the Yukawa coupling of the electron, the two most constraining measurements are the CMS bound on $h \rightarrow e^{+} e^{-}[10]$,

$$
\mu_{e e} \equiv \frac{\sigma(p p \rightarrow h) \mathrm{BR}\left(h \rightarrow e^{+} e^{-}\right)}{\left[\sigma(p p \rightarrow h) \mathrm{BR}\left(h \rightarrow e^{+} e^{-}\right)\right]^{\mathrm{SM}}}<3.7 \times 10^{5},
$$

and the ACME bound on the electron EDM [11],

$$
\left|d_{e}\right|<8.7 \times 10^{-29} \text { e cm. }
$$

With $\kappa_{t} \sim 1$ [12], these bounds translate into [13, 14]

$$
\left|\kappa_{e}\right| \leq 6.1 \times 10^{2}, \quad\left|\tilde{\kappa}_{e}\right| \leq 1.7 \times 10^{-2} .
$$

For the sake of concreteness, we consider the hypothetical case that $\kappa_{e}$ is close to the experimental bound, $\kappa_{e}=\mathcal{O}(500)$.

A significant deviation of $\kappa_{e}$ from unity is most simply accounted for in models with more than one Higgs doublet. Hence, we consider two Higgs doublet models (2HDM). For a review of this framework, see ref. [15]. 2HDM with natural flavor conservation (NFC) predict $\kappa_{e}=\kappa_{\mu}=\kappa_{\tau}$. The measurement of $\mu_{\tau \tau}[2,3]$,

$$
\mu_{\tau \tau}=1.09 \pm 0.23
$$

as well as the upper bound on $\mu_{\mu \mu}[6]$,

$$
\mu_{\mu \mu}<2.8
$$

thus exclude the possibility that $\kappa_{e} \gg 1$ for NFC models. Hence, we consider 2HDM without NFC.

The plan of this paper is as follows. In section 2 we define a basis for the two scalar doublets that is particularly convenient for our purposes. We find conditions under which the Yukawa couplings of fermions to the light scalar $h$, and to the heavy scalars $A, H$ and $H^{ \pm}$, are related to that of the electron. In section 3 we study the implications of a very large or very small $\kappa_{e}$ for the scalar spectrum. In section 4 we obtain constraints on CP violation in the scalar potential when $\kappa_{e}$ is enhanced. We further re-analyze the one-loop contributions to $d_{e}$ for large Yukawa coupling. Section 5 is devoted to discussion of the LHC phenomenology. In section 6 we survey two Higgs doublet models in the literature for which our results provide further insights. We conclude in section 7 . 


\section{Yukawa couplings in 2HDM}

\section{$2.1 \kappa_{e}$ in the " $\beta_{e}$-basis"}

In this section, we assume that $\mathrm{CP}$ is a good symmetry of the scalar potential and of the Yukawa sector. Later we argue that this is actually a requirement (rather than an assumption) if $\kappa_{e} \gg 1$. We use notations and various relations based on ref. [16].

In the Higgs basis, $\left(\Phi_{M}, \Phi_{A}\right)$, defined by

$$
\left\langle\Phi_{M}\right\rangle=v, \quad\left\langle\Phi_{A}\right\rangle=0,
$$

and in the mass basis for the charged leptons, we have

$$
Y^{M}=\operatorname{diag}\left(y_{e}, y_{\mu}, y_{\tau}\right), \quad Y^{A}=\text { arbitrary } .
$$

(Since we deal mostly with the charged lepton sector, we use the notation $Y^{X}$ for the charged lepton Yukawa matrix of $\Phi_{X}$.)

The Yukawa matrices of the neutral CP-even scalars are given by

$$
\begin{aligned}
Y^{h} & =c_{\alpha-\beta} Y^{A}+s_{\beta-\alpha} Y^{M}, \\
Y^{H} & =s_{\alpha-\beta} Y^{A}+c_{\beta-\alpha} Y^{M},
\end{aligned}
$$

where $\alpha-\beta$ is the rotation angle from the $\left(\Phi_{M}, \Phi_{A}\right)$ basis to the $\left(\Phi_{H}, \Phi_{h}\right)$ basis. Here, and in what follows, we use $c_{\phi}, s_{\phi}, t_{\phi}, \cot _{\phi}$ for, respectively, $\sin \phi, \cos \phi, \tan \phi, \cot \phi$. Defining $y_{A}^{e} \equiv Y_{e e}^{A}$, we obtain

$$
\kappa_{e}=s_{\beta-\alpha}+c_{\beta-\alpha}\left(y_{A}^{e} / y_{e}\right) .
$$

Thus

$$
\begin{aligned}
& \left|\kappa_{e}\right| \gg 1 \Longrightarrow\left|c_{\beta-\alpha}\left(y_{A}^{e} / y_{e}\right)\right| \gg 1, \\
& \left|\kappa_{e}\right| \ll 1 \Longrightarrow\left|1+\cot _{\beta-\alpha}\left(y_{A}^{e} / y_{e}\right)\right| \ll 1 .
\end{aligned}
$$

We now rotate to a basis for the scalar doublets, $\left(\Phi_{1}, \Phi_{2}\right)$, that is rotated by an angle $\beta$ from the Higgs basis. We define $y_{1}^{e}=Y_{e e}^{1}$ and $y_{2}^{e}=Y_{e e}^{2}$. We obtain:

$$
\frac{y_{e}^{A}}{y_{e}}=\frac{-s_{\beta} y_{1}^{e}+c_{\beta} y_{2}^{e}}{c_{\beta} y_{1}^{e}+s_{\beta} y_{2}^{e}} .
$$

Things are simplified in a specific basis, where $y_{2}^{e}=0$. We can always find a rotation angle, $\beta=\beta_{e}$ :

$$
\beta_{e}=-\arctan \left(y_{A}^{e} / y_{e}\right),
$$

that takes us to this basis. Then,

$$
\kappa_{e}=s_{\beta-\alpha}-c_{\beta-\alpha} t_{\beta_{e}} .
$$

To have $\left|\kappa_{e}\right| \gg 1$, we need

$$
\left|c_{\beta-\alpha} t_{\beta_{e}}\right| \gg 1 .
$$



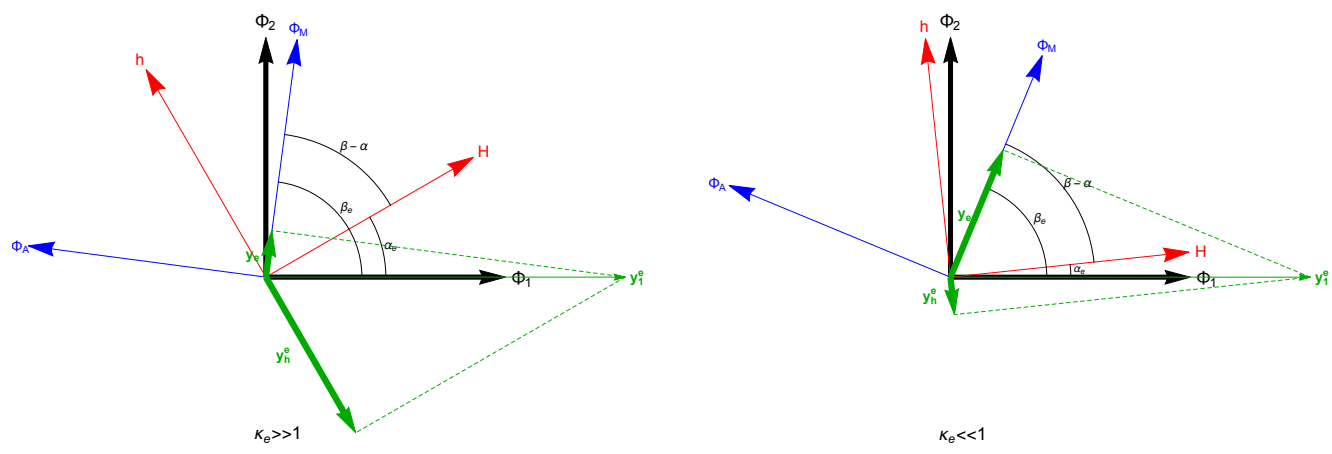

Figure 1. Geometric representation of the three bases, $\left(\Phi_{1}, \Phi_{2}\right)$ (black), $\left(\Phi_{M}, \Phi_{A}\right)$ (blue), and $\left(\Phi_{h}, \Phi_{H}\right)$ (red). Left: $\kappa_{e} \gg 1$. Right: $\kappa_{e} \ll 1$.

To have $\left|\kappa_{e}\right| \ll 1$, we need

$$
\left|\sin \alpha_{e} / \cos \beta_{e}\right| \ll 1,
$$

where $\alpha_{e}$ is the angle $\alpha$, in the basis defined by $\beta_{e}$.

Things are simplified if $\left|c_{\beta-\alpha}\right| \ll 1$ or, in other words, $\kappa_{V} \simeq 1$. In this case we have the following scenarios:

1. $\left|\kappa_{e}\right| \gg 1$ requires $t_{\beta_{e}} \gg t_{\beta-\alpha}: \beta_{e}$ closer to $\pi / 2$ than $\beta-\alpha$ means that $\Phi_{M} \simeq \Phi_{2}$ (the doublet that has no ee coupling).

2. $\left|\kappa_{e}\right| \ll 1$ requires $t_{\beta_{e}} \simeq t_{\beta-\alpha}: \beta_{e}$ close to $\beta-\alpha$ means that $\Phi_{h} \simeq \Phi_{2}$.

3. $\left|\kappa_{e}\right| \simeq 1$ requires $t_{\beta_{e}} \ll t_{\beta-\alpha}$ or $t_{\beta_{e}} \simeq 2 t_{\beta-\alpha}: \beta_{e}$ close to 0 means that $\Phi_{M} \simeq \Phi_{1}$.

The rotations between the three bases that we use for the two Higgs doublets are presented in figure 1.

\section{$2.2 \kappa_{f}$ for $f \neq e$}

In this subsection we identify conditions under which the diagonal couplings of fermions $f \neq e$ are related to that of the electron. For this purpose, we employ the $\beta_{e}$-basis, defined in the previous section. In general, this basis plays no special role for the other fermions, and both $y_{1}^{f}$ and $y_{2}^{f}$ are different from zero. In such a case, there is no further predictive power for $\kappa_{f}$ (unless a specific flavor model is assumed). There are, however, two special cases in which there is a strong predictive power:

1. Similarly to the electron, $y_{2}^{f}=0$. In this case,

$$
\kappa_{f}=\kappa_{e} .
$$

2. In contrast to the electron, $y_{1}^{f}=0$. In this case,

$$
\kappa_{V}=\frac{1+\kappa_{e} \kappa_{f}}{\kappa_{e}+\kappa_{f}} .
$$


In particular, for $\kappa_{e}$ very different from 1 ,

$$
k_{f} \simeq\left\{\begin{array}{cc}
\kappa_{V} & \kappa_{e} \gg 1 \\
\kappa_{V}^{-1} & \kappa_{e} \ll 1 .
\end{array}\right.
$$

Thus, if $y_{2}^{e}=0$ and $y_{1}^{f}=0$, then $\kappa_{e} \gg 1$ or $\ll 1$ implies $\kappa_{f} \approx 1$.

Both of these classes are demonstrated by NFC types II,III,IV. The first case is demonstrated by NFC type I. (See ref. [15] for a review of the various NFC models.) Our findings here are, however, much more general than NFC. They apply whenever, in the fermion mass basis and some basis for the two Higgs doublets, two (or more) diagonal entries vanish. In particular, eqs. (2.11) and (2.12) are independent on whether off-diagonal terms and other diagonal terms vanish (as they do in NFC models) or not.

\section{$2.3 \kappa_{f}^{X}$ for $X=A, H$}

In this subsection we identify conditions under which the diagonal Yukawa couplings of the $H$ and $A$ are related to $Y_{e e}^{h}$. We define

$$
\kappa_{f}^{A, H}=\mathcal{R} e\left(Y_{f f}^{A, H} / y_{f}\right),
$$

For the pseudoscalar $A$, we use the relations between the $\beta_{e}$-basis and the Higgs basis:

$$
\begin{aligned}
Y^{M} & =+c_{\beta_{e}} Y^{1}+s_{\beta_{e}} Y^{2}, \\
Y^{A} & =-s_{\beta_{e}} Y^{1}+c_{\beta_{e}} Y^{2} .
\end{aligned}
$$

For the scalar $H$, we use the relation between the mass basis and the Higgs basis of eq. (2.3), which leads to

$$
\kappa_{f}^{H}=s_{\alpha-\beta} \kappa_{f}^{A}+c_{\alpha-\beta} .
$$

Again, for fermions with $y_{1}^{f} \neq 0$ and $y_{2}^{f} \neq 0$, there is no predictive power for $\kappa_{f}^{A, H}$, but in the other two cases, there is a strong predictive power:

1. $y_{2}^{f}=0$ : using eq. (2.8), we obtain

$$
\begin{aligned}
& \kappa_{f}^{A}=-\tan \beta_{e}=\frac{s_{\alpha-\beta}+\kappa_{e}}{c_{\alpha-\beta}}, \\
& \kappa_{f}^{H}=t_{\alpha-\beta}\left(s_{\alpha-\beta}+\kappa_{e}\right)+c_{\alpha-\beta} .
\end{aligned}
$$

For very large or very small $\kappa_{e}$, we obtain

$$
\begin{aligned}
& k_{f}^{A} \simeq\left\{\begin{array}{ll}
\frac{\kappa_{e}}{c_{\alpha-\beta}} \kappa_{e} \gg 1 \\
t_{\alpha-\beta} & \kappa_{e} \ll 1 .
\end{array},\right. \\
& k_{f}^{H} \simeq\left\{\begin{array}{cc}
t_{\alpha-\beta} \kappa_{e} & \kappa_{e} \gg 1 \\
t_{\alpha-\beta} s_{\alpha-\beta} & \kappa_{e} \ll 1 .
\end{array}\right.
\end{aligned}
$$

Note that these results always apply to $\kappa_{e}^{A, H}$. 
2. $y_{1}^{f}=0$ : using eq. (2.8), we obtain

$$
\begin{aligned}
\kappa_{f}^{A} & =\cot \beta_{e}=\frac{c_{\beta-\alpha}}{s_{\beta-\alpha}-\kappa_{e}}, \\
\kappa_{f}^{H} & =\frac{c_{\alpha-\beta} \kappa_{e}}{s_{\alpha-\beta}+\kappa_{e}} .
\end{aligned}
$$

For very large or very small $\kappa_{e}$, we obtain

$$
\begin{aligned}
& k_{f}^{A} \simeq\left\{\begin{array}{cc}
-\frac{c_{\beta-\alpha}}{\kappa_{e}} & \kappa_{e} \gg 1 \\
t_{\beta-\alpha}^{-1} & \kappa_{e} \ll 1
\end{array},\right. \\
& k_{f}^{H} \simeq\left\{\begin{array}{cc}
c_{\beta-\alpha} & \kappa_{e} \gg 1 \\
\cot _{\alpha-\beta} \kappa_{e} & \kappa_{e} \ll 1 .
\end{array}\right.
\end{aligned}
$$

We learn the following lessons:

- For any fermion with enhanced diagonal Yukawa coupling to the $h$ scalar (such as we assume for the electron in this work), the diagonal Yukawa couplings to the $H$ and $A$ scalars are even further enhanced: $\kappa_{f}^{A, H} / \kappa_{f}=\mathcal{O}\left(t_{\beta-\alpha}\right)$.

- For any fermion with suppressed diagonal Yukawa coupling to the $h$ scalar, the diagonal Yukawa couplings to the $H$ and $A$ scalars are not suppressed, $\kappa_{f}^{A, H}=\mathcal{O}\left(t_{\beta-\alpha}\right)$.

- If, in some basis for the scalar doublets, $Y_{i i}^{2}=0, Y_{j j}^{1}=0$, and $\kappa_{i} \gg 1$, then $\kappa_{j}^{A}$ is highly suppressed and $\kappa_{j}^{H}$ is suppressed but more mildly.

- If, in some basis for the scalar doublets, $Y_{i i}^{1}=0, Y_{j j}^{2}=0$, and $\kappa_{i} \ll 1$, then $\kappa_{j}^{H}$ is highly suppressed and $\kappa_{j}^{A}$ is suppressed but more mildly.

\section{The scalar spectrum}

It is well known that in the decoupling limit of the $2 \mathrm{HDM}, m_{A}^{2} \gg v^{2}$, all the light Higgs boson couplings converge to their SM values. Thus, a large deviation of $\kappa_{e}$ from 1 , the case of interest in this work, seems to require that the second Higgs doublet is not very heavy. In this section we investigate whether there are caveats to this statement, and whether special relations within the scalar spectrum are required for strong enhancement of $\kappa_{e}$. We use the formalism and equations of ref. [17], but apply them specifically in the $\beta_{e}$-basis. Thus, in this section, $\beta$ and $\alpha$ stand for $\beta_{e}$ and $\alpha_{e}$.

The scalar potential is given by

$$
\begin{aligned}
V= & m_{1}^{2}\left(\Phi_{1}^{\dagger} \Phi_{1}\right)+m_{2}^{2}\left(\Phi_{2}^{\dagger} \Phi_{2}\right)-\left[m_{12}^{2}\left(\Phi_{1}^{\dagger} \Phi_{2}\right)+\text { h.c. }\right] \\
& +\frac{\lambda_{1}}{2}\left(\Phi_{1}^{\dagger} \Phi_{1}\right)^{2}+\frac{\lambda_{2}}{2}\left(\Phi_{2}^{\dagger} \Phi_{2}\right)^{2}+\lambda_{3}\left(\phi_{1}^{\dagger} \Phi_{1}\right)\left(\Phi_{2}^{\dagger} \Phi_{2}\right)+\lambda_{4}\left(\phi_{1}^{\dagger} \Phi_{2}\right)\left(\phi_{2}^{\dagger} \Phi_{1}\right) \\
& +\left\{\frac{\lambda_{5}}{2}\left(\Phi_{1}^{\dagger} \Phi_{2}\right)^{2}+\left[\lambda_{6}\left(\Phi_{1}^{\dagger} \Phi_{1}\right)+\lambda_{7}\left(\Phi_{2}^{\dagger} \Phi_{2}\right)\right] \Phi_{1}^{\dagger} \Phi_{2}+\text { h.c. }\right\} .
\end{aligned}
$$


In a general, but $\mathrm{CP}$ conserving, 2HDM, the masses-squared of the CP-odd neutral and the charged scalar are given by

$$
\begin{aligned}
m_{A}^{2} & =\frac{m_{12}^{2}}{s_{\beta} c_{\beta}}-\frac{1}{2} v^{2}\left(2 \lambda_{5}+\lambda_{6} t_{\beta}^{-1}+\lambda_{7} t_{\beta}\right), \\
m_{H^{ \pm}}^{2} & =m_{A}^{2}+\frac{1}{2} v^{2}\left(\lambda_{5}-\lambda_{4}\right) .
\end{aligned}
$$

The mass-squared matrix for the neutral CP-even Higgs bosons, $H$ and $h$, is given by

$$
\begin{aligned}
\mathcal{M}^{2} & =m_{A}^{2}\left(\begin{array}{cc}
s_{\beta}^{2} & -s_{\beta} c_{\beta} \\
-s_{\beta} c_{\beta} & c_{\beta}^{2}
\end{array}\right)+\mathcal{B}^{2} \\
\mathcal{B}^{2} & =v^{2}\left(\begin{array}{ll}
\lambda_{1} c_{\beta}^{2}+2 \lambda_{6} s_{\beta} c_{\beta}+\lambda_{5} s_{\beta}^{2} & \lambda_{34} s_{\beta} c_{\beta}+\lambda_{6} c_{\beta}^{2}+\lambda_{7} s_{\beta}^{2} \\
\lambda_{34} s_{\beta} c_{\beta}+\lambda_{6} c_{\beta}^{2}+\lambda_{7} s_{\beta}^{2} & \lambda_{2} s_{\beta}^{2}+2 \lambda_{7} s_{\beta} c_{\beta}+\lambda_{5} c_{\beta}^{2}
\end{array}\right),
\end{aligned}
$$

where $\lambda_{34} \equiv \lambda_{3}+\lambda_{4}$.

Eq. (2.8) implies that $\kappa_{e} \gg 1$ requires $t_{\beta_{e}} \gg 1$, and that for $c_{\alpha-\beta} \ll 1$ also $\kappa_{e} \ll 1$ requires $t_{\beta_{e}} \gg 1$. We thus take the $t_{\beta} \gg 1$ and $c_{\beta-\alpha} \ll 1$ limits.

\section{$3.1 m_{A}^{2} \gg v^{2}$}

The main question in our mind is whether measuring $\kappa_{e} \gg 1$ will imply that a second Higgs doublet is necessarily within the reach of the LHC experiments. Thus, we expand the above expressions in the $m_{A}^{2} \gg v^{2}$ limit. We obtain the following relation between $\kappa_{e}$ and the scalar related parameters:

$$
\kappa_{V}-\kappa_{e} \simeq \frac{\lambda_{7} v^{2} t_{\beta}}{m_{A}^{2}} .
$$

We learn that there is an interesting range, $v^{2} \ll m_{A}^{2} \ll v^{2} t_{\beta}$, where $\kappa_{e} \gg 1$ is possible, yet the second Higgs doublet is too heavy to be directly produced by the LHC. It is important to remember that $t_{\beta_{e}}$ can be very large. Perturbativity requires that $t_{\beta_{e}} \lesssim 1 / y_{e}$ (four orders of magnitude above the bound in, for example, NFC type-II models, $\left.t_{\beta} \lesssim 1 / y_{b}\right)$. In fact, to achieve $\kappa_{e}=\mathcal{O}(500)$ with $c_{\alpha-\beta} \lesssim 0.1$, we need $t_{\beta_{e}} \gtrsim 5000$.

The larger $m_{A}^{2}$, the smaller $c_{\alpha-\beta} \approx \lambda_{7} v^{2} / m_{A}^{2}$, which leads to larger $y_{e}^{A}$. Requiring that $y_{e}^{A}$ is perturbative implies, for large $\kappa_{e}, m_{A}^{2} \lesssim v^{2} /\left(y_{e} \kappa_{e}\right)$. We conclude that, for large $\kappa_{e}$, $m_{A}^{2}$ can be of $\mathcal{O}\left[v^{2} /\left(y_{e} \kappa_{e}\right)\right] \gg v^{2}$. For $\kappa_{e}=\mathcal{O}(500)$, we can have $m_{A}=\mathcal{O}(10 \mathrm{TeV})$.

It is interesting to understand how this scenario translates into the language of the SM as an effective field theory (EFT). In general, if there are no new light degrees of freedom, modifications of the electron Yukawa coupling come from higher-dimensional operators. Consider the terms

$$
\lambda_{i j} \overline{L_{i}} \phi E_{j}+\frac{\lambda_{i j}^{\prime}}{\Lambda^{2}}\left(\phi^{\dagger} \phi\right) \overline{L_{i}} \phi E_{j}
$$


where $\phi$ is the Higgs doublet, $L_{i}$ are the left-handed lepton doublets and $E_{j}$ are the righthanded charged lepton singlets. Then,

$$
\begin{aligned}
m_{e} & =\frac{v}{\sqrt{2}}\left(\lambda_{e e}+\frac{v^{2} \lambda_{e e}^{\prime}}{2 \Lambda^{2}}\right), \\
Y_{e e}^{h} & =\lambda_{e e}+\frac{3 v^{2} \lambda_{e e}^{\prime}}{2 \Lambda^{2}} .
\end{aligned}
$$

In general, we expect $\kappa_{e}$ to be between 1 , which corresponds to the case that the renormalizable term dominates, and 3 , which corresponds to the case that the dimension-six term dominates. It is possible, however, to have $\kappa_{e} \gg 1$, if $\lambda_{e e}+\left(\lambda_{e e}^{\prime} / 2\right)\left(v^{2} / \Lambda^{2}\right) \ll \lambda_{e e}$. Indeed, this is what is happening in our $2 \mathrm{HDM}$. We obtain, for $m_{11}^{2} \gg m_{12}^{2}, m_{22}^{2}$ and large $\tan \beta_{e}$,

$$
\begin{aligned}
\lambda_{e e} & =\sqrt{2}\left(Y_{e e}^{h}+\frac{Y_{e e}^{H} m_{H h}^{2}}{m_{H H}^{2}}\right), \\
\frac{v^{2} \lambda_{e e}^{\prime}}{2 \Lambda^{2}} & =-\frac{Y_{e e}^{H} \lambda_{H h h h} v^{2}}{\sqrt{2} m_{H H}^{2}} .
\end{aligned}
$$

Here, $m_{H H}^{2}, m_{H h}^{2}$ and $\lambda_{H h h}$ are parameters of the scalar potential in the mass basis $\left(\Phi_{H}, \Phi_{h}\right)$, corresponding to $m_{11}^{2}, m_{12}^{2}$ and $\lambda_{7}$, respectively. The mass basis parameters fulfill $m_{H h}^{2} \approx(3 / 2) \lambda_{H h h h} v^{2}$. Moreover, in the region of interest, $Y_{e e}^{h} / Y_{e e}^{H}=-c_{\beta-\alpha} \approx$ $-\lambda_{H h h} v^{2} / m_{H H}^{2}$. Consequently, the required cancelation occurs.

\section{$3.2 \quad \lambda_{6}=\lambda_{7}=0$}

In various models, zeros in the Yukawa matrices are generated by a $Z_{2}$ symmetry, under which one of the scalar doublets is even and the other is odd. For phenomenological reasons, the symmetry is usually assumed to be softly broken, namely $m_{12}^{2} \neq 0$ while $\lambda_{6}=\lambda_{7}=0$. For this class of models, We obtain the following relation between $\kappa_{e}$ and the scalar related parameters:

$$
\frac{m_{A}^{2}-\lambda_{34} v^{2}}{m_{H}^{2}-m_{h}^{2}}=\frac{\kappa_{e}\left(\kappa_{V} \kappa_{e}-1\right)}{\kappa_{e}-\kappa_{V}} .
$$

We learn the following:

1. $\kappa_{e} \gg 1$ requires

$$
m_{H}^{2} \simeq m_{h}^{2}
$$

2. $\kappa_{e} \ll 1$ requires

$$
m_{A}^{2} \simeq \lambda_{34} v^{2}
$$

In either case, $m_{A}^{2}=\mathcal{O}\left(v^{2}\right)$.

In fact, for $m_{A}^{2} \gg v^{2}$ we have $\kappa_{e}=1+\mathcal{O}\left(v^{2} / m_{A}^{2}\right)$. To understand why this is the case even for $v^{2} \ll m_{A}^{2} \ll v^{2} \tan \beta$, note that, for $m_{A}^{2} \gg v^{2}$ and $\lambda_{6}=\lambda_{7}=0$ [17]:

$$
c_{\beta-\alpha} t_{\beta}=\left(\lambda_{34}+\lambda_{5}-\lambda_{2}\right)\left(v^{2} / m_{A}^{2}\right) .
$$

In other words, in the limit of $m_{A}^{2} \gg v^{2}$ and $\tan \beta \gg 1, \cos (\beta-\alpha)$ is further suppressed below $\left(v^{2} / m_{A}^{2}\right)$, in such a way that $c_{\beta-\alpha} t_{\beta}$ is small, $\mathcal{O}\left(v^{2} / m_{A}^{2}\right)$, and $\kappa_{e}$ is consequently $\mathcal{O}(1)$. 


\section{CP violation}

Given that we consider the hypothetical case of $\kappa_{e} \sim 500$, and that there is a bound on $\tilde{\kappa}_{e}<0.017$ [14], CP must be a very good symmetry (broken at a level smaller than $10^{-4}-10^{-5}$ ) in this context. Here we investigate $\mathrm{CP}$ violation in the scalar potential and in the Yukawa couplings, assuming no cancelations between these two sources of $\tilde{\kappa}_{e} \neq 0$. (For previous, related studies, see refs. [18-23].)

\subsection{The scalar sector}

We use here the formalism of ref. [18]. Consider the scalar potential of eq. (3.1) where, for simplicity, we take $\lambda_{6}=\lambda_{7}=0$. We work in the basis where $v_{1}$ is real. We define

$$
\begin{aligned}
\tan \beta & =\left|v_{2} / v_{1}\right|, \\
\mu_{12} & =\mathcal{R} e\left(m_{12}^{2}\right) /\left(v^{2} c_{\beta} s_{\beta}\right), \\
\lambda_{345} & =\lambda_{3}+\lambda_{4}+\mathcal{R} e\left(\lambda_{5}\right) .
\end{aligned}
$$

The scalar potential gives the following mass-squared matrix in the $\left\{H_{1}^{0}, H_{2}^{0}, A^{0}\right\}$ basis:

$$
\mathcal{M}^{2}=v^{2}\left(\begin{array}{ccc}
\lambda_{1} c_{\beta}^{2}+\mu_{12} s_{\beta}^{2} & \left(\lambda_{345}-\mu_{12}\right) c_{\beta} s_{\beta} & -\frac{1}{2} \mathcal{I} m \lambda_{5} s_{\beta} \\
\left(\lambda_{345}-\mu_{12}\right) c_{\beta} s_{\beta} & \lambda_{2} s_{\beta}^{2}+\mu_{12} c_{\beta}^{2} & -\frac{1}{2} \mathcal{I} m \lambda_{5} c_{\beta} \\
-\frac{1}{2} \mathcal{I} m \lambda_{5} s_{\beta} & -\frac{1}{2} \mathcal{I} m \lambda_{5} c_{\beta} & -\mathcal{R} e \lambda_{5}+\mu_{12}
\end{array}\right)
$$

We define the diagonalizing matrix $R$ :

$$
R \mathcal{M}^{\in} R^{T}=\operatorname{diag}\left(m_{h_{1}}^{2}, m_{h_{2}}^{2}, m_{h_{3}}^{2}\right),
$$

so that

$$
\left(\begin{array}{l}
h_{1} \\
h_{2} \\
h_{3}
\end{array}\right)=R\left(\begin{array}{c}
H_{1}^{0} \\
H_{2}^{0} \\
A^{0}
\end{array}\right)
$$

For fields with no diagonal coupling to $\Phi_{2}$, such as $Y_{e e}^{2}=0$, we have

$$
\kappa_{e}=R_{h 1} / c_{\beta}, \quad \tilde{\kappa}_{e}=-R_{h 3} t_{\beta} .
$$

For fields with no diagonal couplings to $\Phi_{1}, Y_{f f}^{1}=0$, we have

$$
\kappa_{f}=R_{h 2} / s_{\beta}, \quad \tilde{\kappa}_{f}=-R_{h 3} \cot \beta .
$$

We learn that for the electron

$$
\tilde{\kappa}_{e} / \kappa_{e}=\left(R_{h 3} / R_{h 1}\right) \sin \beta \text {. }
$$

In particular, an upper bound on $\tilde{\kappa}_{e} / \kappa_{e}$ translates into an upper bound on $R_{h 3} / R_{h 1}$. 
In the large $\tan \beta$ limit, and assuming that the diagonal terms in $\mathcal{M}^{2}$ are not quasidegenerate, we obtain

$$
\begin{aligned}
R_{21} & \sim \frac{1}{t_{\beta}} \frac{\mu_{12}-\lambda_{345}}{\mu_{12}-\lambda_{2}} \\
R_{23} & \sim \frac{1}{2 t_{\beta}} \frac{\mathcal{I} m \lambda_{5}}{\mu_{12}-\lambda_{2}-\mathcal{R} e \lambda_{5}} \\
R_{13} & \sim \frac{1}{2} \frac{\mathcal{I} m \lambda_{5}}{\mathcal{R} e \lambda_{5}}
\end{aligned}
$$

Identifying $h$ with $h_{2}$, we get

$$
\begin{aligned}
\kappa_{e} & \sim \frac{\mu_{12}-\lambda_{345}}{\mu_{12}-\lambda_{2}}, \\
\tilde{\kappa}_{e} & \sim \frac{(1 / 2) \mathcal{I} m \lambda_{5}}{\mu_{12}-\lambda_{2}-\mathcal{R} e \lambda_{5}}, \\
\frac{\tilde{\kappa}_{e}}{\kappa_{e}} & =\mathcal{O}\left(\frac{\mathcal{I} m \lambda_{5}}{\mu_{12}-\lambda_{345}}\right) .
\end{aligned}
$$

One can also express the results in terms of the two rephasing invariant complex phases:

$$
\begin{aligned}
& \delta_{1}=\arg \left[\lambda_{5}^{*}\left(m_{12}^{2}\right)^{2}\right], \\
& \delta_{2}=\arg \left[\lambda_{5}^{*}\left(m_{12}^{2}\right) v_{1} v_{2}^{*}\right] .
\end{aligned}
$$

The minimum equations relate these two phases, so that only one is independent. For $\left|m_{12}^{2}\right| t_{\beta} \gg v^{2}$ and small phases, we have $\delta_{2} \simeq \delta_{1}$, and

$$
\frac{\tilde{\kappa}_{e}}{\kappa_{e}} \simeq \frac{\left|\lambda_{5}\right| \sin \delta_{1}}{\left|m_{12}^{2} / v^{2}\right| t_{\beta}}
$$

We conclude that the phases in the scalar potential, which can a-priori be $\mathcal{O}(1)$, must be smaller - in the case that $\kappa_{e}=\mathcal{O}\left(10^{2}\right)$ - than $\mathcal{O}\left(10^{-4}\right)$. Let us note that even if $\kappa_{e}=\mathcal{O}(1)$, the scalar potential of a $2 \mathrm{HDM}$ should be CP conserving to the level of $10^{-2}$.

\subsection{The Yukawa sector}

In this subsection we assume that $\mathrm{CP}$ is a good symmetry of the scalar potential, such that the neutral mass eigenstates are the even $(h$ and $H)$ and odd $(A) \mathrm{CP}$ eigenstates. We ask whether strong enhancement of $\kappa_{e}^{X}$ makes the bounds from one-loop contributions to $d_{e}$ competitive with the bounds from two-loop contributions (1.5).

The one-loop flavor-conserving Higgs contribution to the electron EDM is given by (for example, see [23])

$$
d_{e}=-\frac{e y_{e}^{2} \tilde{\kappa}_{e} \kappa_{e}}{(4 \pi)^{2}} \frac{m_{e}}{m_{h}^{2}}\left(\ln \frac{m_{e}^{2}}{m_{h}^{2}}+\frac{7}{6}\right)
$$

Imposing the upper bound on $d_{e}$ of eq. (1.4) [11], we arrive at

$$
\kappa_{e} \tilde{\kappa}_{e} \lesssim 1.1 \times 10^{5} .
$$


Since $\kappa_{e} \tilde{\kappa}_{e} \leq \frac{1}{2}\left(\left|\kappa_{e}\right|^{2}+\left|\tilde{\kappa}_{e}\right|^{2}\right)$, eq. (4.13) is automatically satisfied when the bound on $\mu_{e e}$ of eq. (1.3) [10] is imposed.

As for the contribution of the heavy scalar loops, we have

$$
\begin{aligned}
d_{e}^{H} & =-\frac{e y_{e}^{2} \tilde{\kappa}_{e}^{H} \kappa_{e}^{H}}{(4 \pi)^{2}} \frac{m_{e}}{m_{H}^{2}}\left(\ln \frac{m_{e}^{2}}{m_{H}^{2}}+\frac{7}{6}\right) ; \\
d_{e}^{A} & =\frac{e y_{e}^{2} \tilde{\kappa}_{e}^{A} \kappa_{e}^{A}}{(4 \pi)^{2}} \frac{m_{e}}{m_{A}^{2}}\left(\ln \frac{m_{e}^{2}}{m_{A}^{2}}+\frac{11}{6}\right) ; \\
d_{e}^{H^{ \pm}} & =-\frac{e y_{e}^{2} \tilde{\kappa}_{e}^{A} \kappa_{e}^{A}}{6(4 \pi)^{2}} \frac{m_{e}}{m_{H^{ \pm}}^{2}} .
\end{aligned}
$$

We obtain the following bounds:

$$
\tilde{\kappa}_{e}^{H, A} \kappa_{e}^{H, A} \lesssim 7 \times 10^{6}\left(\frac{m_{H, A}}{1 \mathrm{TeV}}\right)^{2}
$$

In the scenario where the scalar potential is real and CP violation comes from phases in the Yukawa entries, we found that $\kappa_{e}^{H, A}$ and $\tilde{\kappa}_{e}^{H, A}$ are $t_{\beta-\alpha}$ enhanced compared to $\kappa_{e}$ and $\tilde{\kappa}_{e}$. Thus, for $c_{\alpha-\beta} \ll 1$, eq. (4.15) provides stronger bounds than eq. (4.13). However, this constraint competes with the bound coming from the Barr-Zee diagrams [12] only if $0<\left|c_{\beta-\alpha}\right| \lesssim 4 \times 10^{-5}\left(\mathrm{TeV} / m_{S}\right)$.

If the heavy scalars are quasi-degenerate, $m_{H} \approx m_{A} \approx m_{H^{ \pm}}$, and $t_{\beta-\alpha} \gg 1$ so that $\kappa_{e}^{H^{ \pm}}=\kappa_{e}^{A} \approx \kappa_{e}^{H} \approx t_{\beta-\alpha} k_{e}$, the total contribution of scalars at one loop is given by

$$
d_{e} \approx-\frac{e y_{e}^{2} \kappa_{e} \tilde{\kappa}_{e}}{(4 \pi)^{2}} \frac{m_{e}}{m_{h}^{2}}\left(\ln \frac{m_{e}^{2}}{m_{h}^{2}}+\frac{7}{6}-\frac{t_{\beta-\alpha}^{2}}{2} \frac{m_{h}^{2}}{m_{H}^{2}}\right) .
$$

\section{LHC phenomenology}

In section 3 we argued that in most of the parameter space relevant to $\kappa_{e} \gg 1$, all scalars should be at the electroweak scale. An exception arises if $\lambda_{7}=\mathcal{O}(1)$, but even in this case a large portion of the parameter space is within the reach of the LHC. Hence, we can probe the $\kappa_{e} \gg 1$ scenario in $2 \mathrm{HDM}$ indirectly via LHC searches for new scalars.

\section{1 $A^{0}, H^{0}$ and $H^{ \pm}$decay modes}

The Yukawa coupling of $A$ to electrons (2.17) is enhanced. In order to establish if the $A \rightarrow e^{+} e^{-}$decay has a phenomenological impact we need to compare eq. (2.17) with the coupling to other SM fermions:

$$
\frac{y_{e}^{A}}{y_{f}^{A}}=\frac{y_{e}}{y_{f}} \frac{\kappa_{e}^{A}}{\kappa_{f}^{A}} .
$$

Our first observation in this regard is that, for $\kappa_{e}=\mathcal{O}(500), A \rightarrow e^{+} e^{-}$will dominate over $A \rightarrow f \bar{f}$ for any fermion $f$ for which $y_{1}^{f}=0$. For such fermions,

$$
\frac{y_{e}^{A}}{y_{f}^{A}}=\frac{y_{e}}{y_{f}} t_{\beta_{e}}^{2}
$$


The strongest hierarchy of the SM Yukawa couplings is for $y_{e} / y_{t}$. If $y_{1}^{t}=0$, then

$$
t_{\beta_{e}} \gtrsim 500 \Longrightarrow y_{e}^{A} \gtrsim y_{t}^{A} \text {. }
$$

This condition is met when $\kappa_{e} \gtrsim 500 \sqrt{1-\kappa_{V}^{2}}$ and, in particular, for $\kappa_{e} \gtrsim 500$, as we assume. Obviously, if $y_{1}^{t}=0$ implies $y_{e}^{A}>y_{t}^{A}$, then $y_{1}^{f}=0$ guarantees $y_{e}^{A}>y_{f}^{A}$ for any fermion $f$. Similar conclusions hold for $H^{+} \rightarrow e^{+} \nu$ and $H^{0} \rightarrow e^{+} e^{-}$.

Our second observation is that, the ratio of $\Gamma\left(A \rightarrow e^{+} e^{-}\right) / \Gamma(A \rightarrow f \bar{f})=\left(y_{e} / y_{f}\right)^{2}$ for any fermion $f$ for which $y_{2}^{f}=0$. Thus, if any second generation fermion (or, obviously, third) has $y_{2}^{f}=0, A \rightarrow e^{+} e^{-}$will have little phenomenological impact. Similar conclusions hold for $H^{+} \rightarrow e^{+} \nu$ and $H^{0} \rightarrow e^{+} e^{-}$. If the $u$-quark and/or the $d$-quark have $y_{2}^{f}=0$, then the dielectron decay rate of the heavy scalars will be subdominant to the dijet rate, but not negligible.

Our third observation makes use of eq. (2.3), which gives

$$
\frac{\kappa_{e}^{A}}{\kappa_{f}^{A}}=\frac{\kappa_{e}-s_{\beta-\alpha}}{\kappa_{f}-s_{\beta-\alpha}} .
$$

Thus, if experiments put an upper bound on $\kappa_{f}, \kappa_{f}^{\max }$, then for large $\kappa_{e} \gg \kappa_{f}^{\max }$, we have

$$
\frac{\kappa_{e}^{A}}{\kappa_{f}^{A}} \gtrsim \frac{\kappa_{e}}{\kappa_{f}^{\max }} .
$$

Such upper bounds apply already to $f=t, b, \tau, c, \mu$. They prove that, if $\kappa_{e}=\mathcal{O}(500)$ then $y_{2}^{f} \neq 0$ for all of these fermions. For the muon case, the present bound is sufficient to guarantee that if $\kappa_{e}=\mathcal{O}(500)$ then $A \rightarrow e^{+} e^{-}$dominates over $A \rightarrow \mu^{+} \mu^{-}$.

\subsection{Multi-electron signatures}

In the previous subsection we obtained conditions under which the $A, H \rightarrow e^{+} e^{-}$and $H^{+} \rightarrow e^{+} \nu$ are the dominant decay modes of the heavy scalars. Specifically, it is required that the heavy scalar couplings to the third generation fermions are strongly suppressed (which is the case for $y_{1}^{f}=0$ ). The conditions for suppressing the heavy scalar decays into third generation fermions also entail strong suppression of single heavy scalar production, e.g. $g g \rightarrow A$ and $g b \rightarrow t H^{-}$. Furthermore, in the $\kappa_{V} \rightarrow 1$ limit, also the production via vector boson fusion is subdominant.

We distinguish two scenarios with large $\kappa_{e}$ : one where $y_{2}^{e}=y_{2}^{d}=y_{2}^{u}=0$ and consequently also $\kappa_{u}, \kappa_{d}$ are large, which we discuss in the next subsection, and one where only $y_{2}^{e}=0$, which we focus on here. In this case, the relevant production modes of heavy scalars are pair production via the $s$-channel mediation of a virtual electroweak vector boson:

$$
p p \rightarrow \gamma^{*} \rightarrow H^{+} H^{-}, \quad p p \rightarrow W^{*} \rightarrow H^{+} A^{0}\left(H^{0}\right), \quad p p \rightarrow Z^{*} \rightarrow A^{0} H^{0} .
$$

Since we assume here that the branching ratio into electrons is dominant, the relevant topologies are the following: 
- Two electrons plus missing energy:

$$
p p \rightarrow \gamma^{*} / Z^{*} \rightarrow H^{+} H^{-} \rightarrow e^{+} e^{-} \nu \nu .
$$

- Three electron plus missing energy:

$$
p p \rightarrow W^{*} \rightarrow H^{0}\left(A^{0}\right) H^{ \pm} \rightarrow e^{+} e^{-} e^{ \pm} \nu .
$$

- Four electrons:

$$
p p \rightarrow Z^{*} \rightarrow H^{0} A^{0} \rightarrow e^{+} e^{-} e^{+} e^{-}
$$

Hence multi-electron signatures are the distinctive feature of this scenario.

We are not aware of relevant targeted searches for this topology in 2HDM. However, multi-lepton searches, typically aimed for models of neutrino masses, have been carried out and can be recasted for our topology. In particular, the $13 \mathrm{TeV}$ CMS search [24] is a multipurpose analysis which can be applied to other topologies other than the one originally designed for. A recast was made in ref. [25] assuming dominance of final multi-muon states and $s_{\beta-\alpha}=1$, and a bound $m_{A}>640 \mathrm{GeV}$ was extracted. ${ }^{1}$ Since we do not expect the efficiency to change significantly, we take this bound to be a rough indication of the bound that applies for the multi-electron case. We conclude:

- The scenario with $\lambda_{6,7}=0$, where $\kappa_{e} \gg 1$ requires $m_{H} \simeq m_{h}$ [see eq. (3.9)], is excluded;

- The scenario with hard breaking of the $Z_{2}$ symmetry is strongly constrained unless $\lambda_{7}$ is very large or $\kappa_{V}$ very close to 1 .

\subsection{Large first generation Yukawa couplings}

An interesting case arises if one of the Higgs doublets does not couple to the first generation fermions, $y_{2}^{e}=y_{2}^{d}=y_{2}^{u}=0$ and, consequently, $\kappa_{e}=\kappa_{d}=\kappa_{u}$. In this case, the branching ratio of the heavy scalars to dielectrons is considerably smaller than the branching ratio into dijets, but the production cross-section is enhanced via the $s$-channel $q \bar{q}^{\prime} \rightarrow A, H, H^{ \pm}$. Therefore, dielectron resonance searches can become relevant.

In order to explore the phenomenological implications of such a framework, we further assume that, to a good approximation, the other Higgs doublet does not couple to the second and third generations. Thus, we consider the following scenario:

$$
\begin{aligned}
& \kappa_{e}=\kappa_{u}=\kappa_{d}, \\
& \kappa_{t}=\kappa_{b}=\kappa_{\tau}=\kappa_{c}=\kappa_{s}=\kappa_{\mu},
\end{aligned}
$$

and

$$
\kappa_{V}=\frac{1+\kappa_{e} \kappa_{t}}{\kappa_{e}+\kappa_{t}}
$$

\footnotetext{
${ }^{1}$ For $0.9<s_{\beta-\alpha}<1$ the bound does not change significantly, hence we can consider this as the reference value.
} 


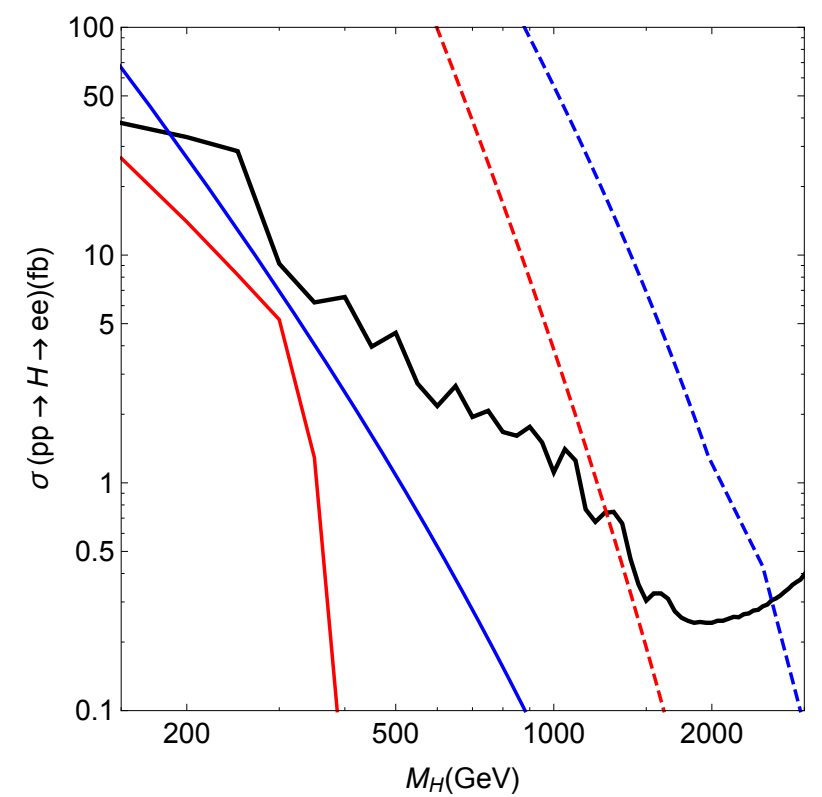

Figure 2. $\sigma\left(p p \rightarrow H, A \rightarrow e^{+} e^{-}\right)$as a function of $m_{H}=m_{A}$ for $\kappa_{e}=500$. The region above the black curve is excluded by the $8 \mathrm{TeV}$ ATLAS search [26]. The theoretical predictions corresponding to the scenario defined by eqs. (5.10) and (5.11) are given in blue curves, with $t_{\beta}=1000$ (solid) or $4 \times 10^{4}$ (dashed). The theoretical predictions corresponding to $y_{t}^{A, H}=1$ are given in red curves, with $\tan \beta=1000$ (solid) or $4 \times 10^{5}$ (dashed).

Therefore, the couplings of the extra scalars to the whole first generation are $t_{\beta}$ enhanced, so this is the relevant parameter to evaluate the constraints, together with the mass $m_{H}$.

The mass window 120-150 GeV is probed by the CMS search for $h \rightarrow e^{+} e^{-}[10]$ and is excluded for $t_{\beta}>800-900$. For $\kappa_{V} \sim 1$ this implies that also moderate values for $\kappa_{e, u, d}$ are ruled out.

Heavier masses are constrained by dilepton searches from both ATLAS [26] and CMS [27]. These searches look for both dielectron and dimuon final states, targeting as benchmark models new $Z^{\prime}$ gauge bosons. Their limit can be straightforwardly applied to our scenario, as long as they are presented separately for dimuons and dielectrons. We obtain the exclusion limits on our scenario from the $8 \mathrm{TeV}$ data. The current $13 \mathrm{TeV}$ data do not change the picture significantly. CMS published so far results with only $2.9 \mathrm{fb}^{-1}$ data from run II [28], while ATLAS [29] present only combined results from dielectron and dimuon channels.

Figure 2 presents the constraints from resonant dielectron $8 \mathrm{TeV}$ searches in the $150 \mathrm{GeV}-3 \mathrm{TeV}$ range. The experimental exclusion curve, based on [26], is given in solid black (similar results follow from the CMS search [27]). We computed the leading order cross-section for $q \bar{q}^{\prime} \rightarrow H, A \rightarrow e^{+} e^{-}$using the NNPDF2.3 LO pdf Mathematica package $[30,31]$. We use two values for $t_{\beta}$ : first, $t_{\beta}=1000$ (solid blue curve), which is close to the minimum value constrained by this search. Second, $t_{\beta}=4 \times 10^{4}$ (dashed blue curve), which corresponds to $1 / y_{d}$, the maximum allowed value from perturbativity. 
We reach the following conclusions:

- The CMS search [10] rules out the existence of $H^{0}$ and $A^{0}$ with mass in the $120-150 \mathrm{GeV}$ range and $\tan \beta>900$. For $\kappa_{e}=500$, this implies in turn $\kappa_{V}>0.83$. (For $\kappa_{e}=50$, this implies $\kappa_{V}>0.998$ ).

- The ATLAS search [26] rules out $m_{H, A}<200$ (2500) GeV for $\tan \beta>10^{3}\left(1 / y_{d}\right)$.

Hence, the scenario with $\lambda_{6,7}=0$ is almost ruled out for all values of $t_{\beta}$. We expect in the future the limit in the high $t_{\beta}$ to become more stringent pushing towards higher values of $\lambda_{7}$ or $\kappa_{V}$ closer to one.

\subsection{Production via gluon gluon fusion}

Another scenario which gives rise to resonant production via gluon gluon fusion of the extra scalars and subsequent decay into $e^{+} e^{-}$corresponds to $y_{1}^{t, b} \neq 0$. As a case study we consider the following benchmark point:

$$
\kappa_{t}^{A, H}=\kappa_{b}^{A, H}=\kappa_{\tau}^{A, H}=1, \quad \kappa_{e}^{A, H}=t_{\beta} .
$$

As concerns $A$ and $H$ production, only $\kappa_{t}^{A, H}$ is relevant. As concerns the decay, especially for $m_{H, A}<2 m_{t}$, also $\kappa_{\tau, b}^{A, H}$ play a role. We consider then the same LHC searches described in the previous subsection. We compute the cross section also with the NNPDF2.3 LO pdf Mathematica package [30,31]. The results are presented in figure 2 in red curves for $t_{\beta}=1000$ (solid) and $t_{\beta}=1 / y_{e}$ (dashed). We reach the following conclusions:

- The searches do not constrain this scenario in the "low" $\tan \beta$ region $\left(t_{\beta}<1000\right)$ in both the low mass [10] and the high mass [26] ranges. (We do expect mild constraints from present searches when NLO corrections are incorporated.)

- The large $\tan \beta$ region, $t_{\beta} \sim 1 / y_{e}$, is excluded up to $m_{H, A} \sim 1.8 \mathrm{TeV}$.

This scenario is then less constrained compared to the previous one. The reason is that the production cross section does not increase with $t_{\beta}$, but only the branching ratio which is, however, very suppressed when the decay mode into two tops is open.

\subsection{Future prospects}

Various suggestions have been made and explored how to further probe the Higgs couplings to the light fermions. It will be possible to improve the constraint on $\kappa_{e}$ by resonant Higgs production at the next-generation high-luminosity $e^{+} e^{-}$collider $[14,32]$ and on $\tilde{\kappa}_{e}$ by future EDM measurements [14]. Ref. [33] suggests to constrain $\kappa_{s}$ via measurements of $h \rightarrow \phi \gamma$, and $\kappa_{u, d}$ via measurements of $h \rightarrow(\rho, \omega) \gamma$. The ATLAS collaboration has recently searched for these decays [34], and a study of the prospects of such measurements in the FCC can be found in ref. [32], Additional ways to probe the Higgs couplings to light quarks include Higgs kinematics [35, 36], hadronic event shapes [37], the $W^{ \pm} h$ charge asymmetry [38], and Higgs decays to light (untagged) jets [39]. Finally, ref. [40] points out that, if Higgs-portal dark matter is discovered, direct searches for dark matter could lead to interesting bounds on the light-quark Yukawa couplings. 


\section{$6 \quad 2 \mathrm{HDM}$ in the literature}

Some of our results have implications that are generic to $2 \mathrm{HDM}$, and go beyond the specific scenario of $\kappa_{e} \gg 1$ or $\kappa_{e} \ll 1$. We here give a brief survey of models that have been proposed in the literature, and their relation with our findings.

\subsection{Separating the third generation from the first two}

In the $2 \mathrm{HDM}$ of ref. [8], one of the Higgs doublets does not couple to the third generation quarks, while the other has negligibly small diagonal couplings to the first two generations. (See ref. [41] for a related scenario.) Thus, in this model,

$$
\begin{aligned}
\kappa_{l} & \equiv \kappa_{u}=\kappa_{d}=\kappa_{s}=\kappa_{c}, \\
\kappa_{h} & \equiv \kappa_{b}=\kappa_{t},
\end{aligned}
$$

and

$$
\kappa_{V}=\frac{1+\kappa_{l} \kappa_{h}}{\kappa_{l}+\kappa_{h}}
$$

The authors aim to have $\kappa_{l} \ll 1$. Given that it is experimentally known that both $\kappa_{V}$ and $\kappa_{h}$ are $\mathcal{O}(1)$ then, in this case, the model predicts $\kappa_{V} \kappa_{h} \simeq 1$.

The model further has $\lambda_{6}=\lambda_{7}=0$ and is thus subject to the analysis of section 3.2. The model however further assumes $\lambda_{3}=\lambda_{4}=\lambda_{5}=0$. The requirement for $\kappa_{e} \gg 1$ is still (3.9), but the requirement for $\kappa_{e} \ll 1$ is no longer (3.10). Examining eq. (3.8), we learn that $\kappa_{e} \ll 1$ implies $\kappa_{e} / \kappa_{V} \simeq m_{A}^{2} /\left(m_{H}^{2}-m_{h}^{2}\right)$, so that $m_{A}^{2} \ll m_{H}^{2}-m_{h}^{2}$ is required. Since in this case

$$
m_{H}^{2}+m_{h}^{2}=m_{A}^{2}+v^{2}\left(\lambda_{1} c_{\beta}^{2}+\lambda_{2} s_{\beta}^{2}\right),
$$

we must have $m_{H}^{2}=\mathcal{O}\left(v^{2}\right)$ and $m_{A}^{2} \ll v^{2}\left(\lambda_{1} c_{\beta}^{2}+\lambda_{2} s_{\beta}^{2}\right)$. We learn that the scalar spectrum is light. A problem might arise however given that for $\lambda_{4}=\lambda_{5}=0$ we have $m_{H^{ \pm}}^{2}=m_{A}^{2}$, and there is a rather strong lower bound on $m_{H^{ \pm}}^{2}$.

In the $2 \mathrm{HDM}$ of ref. [9], one of the Higgs doublets, $\phi$, does not couple to the first two generations, while the other, $\phi^{\prime}$, has small couplings to the third generation. Thus, in this model, $y_{\phi}^{e}=y_{\phi}^{\mu}=0$, and the model should have $\kappa_{e}=\kappa_{\mu}$. Various equations of ref. [9] can be evaluated to get eq. (2.12) as an approximate relation.

In the 2HDM of ref. [7], the masses of the first two quark generations come from dimension-six terms. Thus, the model predicts

$$
\kappa_{c}=\kappa_{s}=\kappa_{d}=\kappa_{u}=3 .
$$

\subsection{Separating the muon from the other fermions}

In the $\mu 2 \mathrm{HDM}$ of ref. [25], one of the Higgs doublets, $H_{2}$, couples to the up sector, to the down sector, and to $e$ and $\tau$. The other Higgs doublet, $H_{1}$, couples to only $\mu$. Thus, for the quarks, this is an NFC model, with the well known consequences of that. In the charged lepton sector, however, we have the situation where

$$
y_{2}^{\mu}=0, \quad y_{1}^{e}=y_{1}^{\tau}=0
$$


From the discussion in section 2.2, the following relations hold:

$$
\kappa_{\tau}=\kappa_{e}, \quad \kappa_{V}=\frac{1+\kappa_{\mu} \kappa_{\tau}}{\kappa_{\mu}+\kappa_{\tau}} .
$$

Thus, the experimental information that $\kappa_{V}$ and $\kappa_{\tau}$ are close to 1 , implies that so is not only $\kappa_{e}$ but also $\kappa_{\mu}$.

\section{Conclusions}

We studied the implications of a strongly enhanced Higgs-electron Yukawa coupling, such that $h \rightarrow e^{+} e^{-}$will be within reach of ATLAS/CMS in the near future, $\kappa_{e} \equiv Y_{e e} / Y_{e e}^{\mathrm{SM}}=$ $\mathcal{O}(500)$. We focussed on two Higgs doublet models (2HDM). Given the experimental measurements of $h \rightarrow \tau^{+} \tau^{-}$and the upper bound on $h \rightarrow \mu^{+} \mu^{-}$, such a strong enhancement of $Y_{e e}$ excludes also $2 \mathrm{HDM}$ with natural flavor conservation (NFC). We thus explored generic $2 \mathrm{HDM}$.

We suggested a basis for the two scalar doublets which is particularly convenient to study implications of enhanced electron Yukawa coupling. Our proposed basis can be straightforwardly generalized to any other fermion with a Yukawa coupling that is very different from the SM prediction.

Our main findings are the following:

- Case I: for two fermions with vanishing Yukawa couplings to one and the same of the two Higgs doublets, the enhancement factors are the same, $\kappa_{f_{1}}=\kappa_{f_{2}}$. Furthermore, the modification factors of their couplings to the heavy scalars, $H, A, H^{ \pm}$, are the same.

- Case II: for two fermions with vanishing Yukawa couplings to two different Higgs doublets, the enhancement factors fulfill a relation, $\kappa_{V}=\left(1+\kappa_{f_{1}} \kappa_{f_{2}}\right) /\left(\kappa_{f_{1}}+\kappa_{f_{2}}\right)$. Similarly, their couplings to heavy scalars fulfill predictive relations.

- If the Yukawa coupling to the Higgs is enhanced, $Y_{f f}^{h} \gg \sqrt{2} m_{f} / v$, the Yukawa coupling to the heavy scalars is even more strongly enhanced (by order $\tan (\alpha-\beta)$ ).

- In case II, if $\kappa_{f_{1}} \gg 1$ so that the Yukawa couplings of $f_{1}$ to $A, H, H^{ \pm}$are very large, $\kappa_{f_{2}} \approx 1$ while the couplings of $f_{2}$ to the heavy scalars are suppressed.

- In models with only soft breaking of a $Z_{2}$ symmetry in the scalar potential, a large deviation of $\kappa_{e}$ from 1 requires a light scalar spectrum. With hard breaking, there is an interesting range where $v^{2} \ll m_{A}^{2} \ll v^{2} \kappa_{e} / \sqrt{1-\kappa_{V}^{2}}$ where such deviation is still possible.

- For $\kappa_{e} \gtrsim 1 \mathrm{CP}$ symmetry should hold to a good approximation $\left[\mathcal{O}\left(10^{-2} / \kappa_{e}\right)\right]$ in both the scalar potential and the Yukawa couplings.

- Large regions of the parameter space of $2 \mathrm{HDM}$ models with $\kappa_{e} \gg 1$ are probed by ATLAS/CMS searches for deviations of the $e^{+} e^{-}$mass spectra from the SM.

- Searches for $e^{+} e^{-} e^{+} e^{-}$and $e^{+} e^{-} e^{ \pm} / E_{T}$ topologies will provide sensitive probes of this scenario. 
We believe that an experimental search for $h \rightarrow e^{+} e^{-}$is a worthy effort, in spite of the gap between the SM prediction and the experimental reach, with the following points in mind:

- The present upper bound, weak as it is, is already excluding universality between the tau and electron Yukawa couplings. If experiments can establish that the electron Yukawa is smaller than the muon Yukawa, it would provide an important input for flavor physics.

- The possibility that $\kappa_{e}$ is close to the experimental bound is not inconsistent with any indirect constraint.

- On the theoretical side, a strong fine-tuning is required, of order $1 / \kappa_{e}$, for real parameters in the scalar potential, and order $10^{-2} / \kappa_{e}$ for complex phases.

- The strong deviation of $\kappa_{e}$ from unity does not guarantee that the second Higgs doublet is light enough to be directly produced at the LHC.

\section{Acknowledgments}

We thank João Silva and Ryosuke Sato for useful discussions. YN is the Amos de-Shalit chair of theoretical physics. YN is supported by grants from the Israel Science Foundation (grant number 394/16), the United States-Israel Binational Science Foundation (BSF), Jerusalem, Israel (grant number 2014230), the I-CORE program of the Planning and Budgeting Committee and the Israel Science Foundation (grant number 1937/12), and the Minerva Foundation.

Open Access. This article is distributed under the terms of the Creative Commons Attribution License (CC-BY 4.0), which permits any use, distribution and reproduction in any medium, provided the original author(s) and source are credited.

\section{References}

[1] ATLAS collaboration, Evidence for the $H \rightarrow b \bar{b}$ decay with the ATLAS detector, JHEP 12 (2017) 024 [arXiv: 1708.03299] [INSPIRE].

[2] CMS collaboration, Observation of the Higgs boson decay to a pair of $\tau$ leptons with the CMS detector, Phys. Lett. B 779 (2018) 283 [arXiv:1708.00373] [INSPIRE].

[3] ATLAS collaboration, Evidence for the Higgs-boson Yukawa coupling to tau leptons with the ATLAS detector, JHEP 04 (2015) 117 [arXiv: 1501.04943] [INSPIRE].

[4] ATLAS, CMS collaborations, Measurements of the Higgs boson production and decay rates and constraints on its couplings from a combined ATLAS and CMS analysis of the LHC pp collision data at $\sqrt{s}=7$ and $8 \mathrm{TeV}, J H E P 08$ (2016) 045 [arXiv: 1606.02266] [INSPIRE].

[5] ATLAS collaboration, Search for the Decay of the Higgs Boson to Charm Quarks with the ATLAS Experiment, arXiv:1802.04329 [INSPIRE]. 
[6] ATLAS collaboration, Search for the dimuon decay of the Higgs boson in pp collisions at $\sqrt{s}=13 \mathrm{TeV}$ with the ATLAS detector, Phys. Rev. Lett. 119 (2017) 051802 [arXiv: 1705.04582] [INSPIRE].

[7] F.J. Botella, G.C. Branco, M.N. Rebelo and J.I. Silva-Marcos, What if the masses of the first two quark families are not generated by the standard model Higgs boson?, Phys. Rev. D 94 (2016) 115031 [arXiv:1602.08011] [InSPIRE].

[8] D. Ghosh, R.S. Gupta and G. Perez, Is the Higgs Mechanism of Fermion Mass Generation a Fact? A Yukawa-less First-Two-Generation Model, Phys. Lett. B 755 (2016) 504 [arXiv: 1508.01501] [INSPIRE].

[9] W. Altmannshofer, S. Gori, A.L. Kagan, L. Silvestrini and J. Zupan, Uncovering Mass Generation Through Higgs Flavor Violation, Phys. Rev. D 93 (2016) 031301 [arXiv: 1507.07927] [INSPIRE].

[10] CMS collaboration, Search for a standard model-like Higgs boson in the $\mu^{+} \mu^{-}$and $e^{+} e^{-}$ decay channels at the LHC, Phys. Lett. B 744 (2015) 184 [arXiv:1410.6679] [InSPIRE].

[11] ACME collaboration, J. Baron et al., Order of Magnitude Smaller Limit on the Electric Dipole Moment of the Electron, Science 343 (2014) 269 [arXiv:1310.7534] [INSPIRE].

[12] S.M. Barr and A. Zee, Electric Dipole Moment of the Electron and of the Neutron, Phys. Rev. Lett. 65 (1990) 21 [Erratum ibid. 65 (1990) 2920] [INSPIRE].

[13] J. Brod, U. Haisch and J. Zupan, Constraints on CP-violating Higgs couplings to the third generation, JHEP 11 (2013) 180 [arXiv:1310.1385] [INSPIRE].

[14] W. Altmannshofer, J. Brod and M. Schmaltz, Experimental constraints on the coupling of the Higgs boson to electrons, JHEP 05 (2015) 125 [arXiv: 1503.04830] [INSPIRE].

[15] G.C. Branco, P.M. Ferreira, L. Lavoura, M.N. Rebelo, M. Sher and J.P. Silva, Theory and phenomenology of two-Higgs-doublet models, Phys. Rept. 516 (2012) 1 [arXiv:1106.0034] [INSPIRE].

[16] A. Dery, A. Efrati, G. Hiller, Y. Hochberg and Y. Nir, Higgs couplings to fermions: 2HDM with MFV, JHEP 08 (2013) 006 [arXiv:1304.6727] [INSPIRE].

[17] J.F. Gunion and H.E. Haber, The CP conserving two Higgs doublet model: The Approach to the decoupling limit, Phys. Rev. D 67 (2003) 075019 [hep-ph/0207010] [INSPIRE].

[18] S. Inoue, M.J. Ramsey-Musolf and Y. Zhang, CP-violating phenomenology of flavor conserving two Higgs doublet models, Phys. Rev. D 89 (2014) 115023 [arXiv:1403.4257] [INSPIRE].

[19] V.D. Barger, A.K. Das and C. Kao, The Electric dipole moment of the muon in a two-Higgs doublet model, Phys. Rev. D 55 (1997) 7099 [hep-ph/9611344] [INSPIRE].

[20] S. Weinberg, Unitarity Constraints on CP Nonconservation in Higgs Exchange, Phys. Rev. D 42 (1990) 860 [INSPIRE].

[21] G. Blankenburg, J. Ellis and G. Isidori, Flavour-Changing Decays of a $125 \mathrm{GeV}$ Higgs-like Particle, Phys. Lett. B 712 (2012) 386 [arXiv:1202.5704] [INSPIRE].

[22] R. Harnik, J. Kopp and J. Zupan, Flavor Violating Higgs Decays, JHEP 03 (2013) 026 [arXiv: 1209.1397] [INSPIRE].

[23] A. Broggio, E.J. Chun, M. Passera, K.M. Patel and S.K. Vempati, Limiting two-Higgs-doublet models, JHEP 11 (2014) 058 [arXiv:1409.3199] [INSPIRE]. 
[24] CMS collaboration, Search for Evidence of the Type-III Seesaw Mechanism in Multilepton Final States in Proton-Proton Collisions at $\sqrt{s}=13$ TeV, Phys. Rev. Lett. 119 (2017) 221802 [arXiv: 1708.07962] [INSPIRE].

[25] T. Abe, R. Sato and K. Yagyu, Muon specific two-Higgs-doublet model, JHEP 07 (2017) 012 [arXiv:1705.01469] [INSPIRE].

[26] ATLAS collaboration, Search for high-mass dilepton resonances in pp collisions at $\sqrt{s}=8 \mathrm{TeV}$ with the ATLAS detector, Phys. Rev. D 90 (2014) 052005 [arXiv:1405.4123] [INSPIRE].

[27] CMS collaboration, Search for physics beyond the standard model in dilepton mass spectra in proton-proton collisions at $\sqrt{s}=8 \mathrm{TeV}$, JHEP 04 (2015) 025 [arXiv:1412.6302] [INSPIRE].

[28] CMS collaboration, Search for narrow resonances in dilepton mass spectra in proton-proton collisions at $\sqrt{s}=13$ TeV and combination with 8 TeV data, Phys. Lett. B 768 (2017) 57 [arXiv: 1609.05391] [INSPIRE].

[29] ATLAS collaboration, Search for new high-mass phenomena in the dilepton final state using $36 \mathrm{fb}^{-1}$ of proton-proton collision data at $\sqrt{s}=13$ TeV with the ATLAS detector, JHEP 10 (2017) 182 [arXiv: 1707.02424] [INSPIRE].

[30] R.D. Ball et al., Parton distributions with LHC data, Nucl. Phys. B 867 (2013) 244 [arXiv: 1207.1303] [INSPIRE].

[31] N.P. Hartland and E.R. Nocera, A Mathematica interface to NNPDFs, arXiv:1209.2585 [INSPIRE].

[32] D. d'Enterria, Higgs physics at the Future Circular Collider, PoS(ICHEP2016) 434 [arXiv: 1701.02663] [INSPIRE].

[33] A.L. Kagan, G. Perez, F. Petriello, Y. Soreq, S. Stoynev and J. Zupan, Exclusive Window onto Higgs Yukawa Couplings, Phys. Rev. Lett. 114 (2015) 101802 [arXiv:1406.1722] [INSPIRE].

[34] ATLAS collaboration, Search for exclusive Higgs and $Z$ boson decays to $\phi \gamma$ and $\rho \gamma$ with the ATLAS detector, arXiv: 1712.02758 [INSPIRE].

[35] Y. Soreq, H.X. Zhu and J. Zupan, Light quark Yukawa couplings from Higgs kinematics, JHEP 12 (2016) 045 [arXiv: 1606.09621] [INSPIRE].

[36] J. Cohen, S. Bar-Shalom, G. Eilam and A. Soni, Light-quarks Yukawa couplings and new

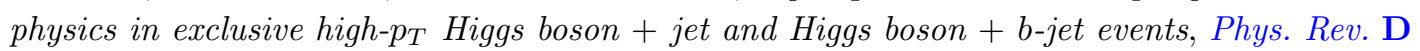
97 (2018) 055014 [arXiv: 1705.09295] [INSPIRE].

[37] J. Gao, Probing light-quark Yukawa couplings via hadronic event shapes at lepton colliders, JHEP 01 (2018) 038 [arXiv: 1608.01746] [INSPIRE].

[38] F. Yu, Phenomenology of Enhanced Light Quark Yukawa Couplings and the $W^{ \pm} h$ Charge Asymmetry, JHEP 02 (2017) 083 [arXiv: 1609.06592] [INSPIRE].

[39] L.M. Carpenter, T. Han, K. Hendricks, Z. Qian and N. Zhou, Higgs Boson Decay to Light Jets at the LHC, Phys. Rev. D 95 (2017) 053003 [arXiv:1611.05463] [INSPIRE].

[40] F. Bishara, J. Brod, P. Uttayarat and J. Zupan, Nonstandard Yukawa Couplings and Higgs Portal Dark Matter, JHEP 01 (2016) 010 [arXiv:1504.04022] [INSPIRE].

[41] W. Altmannshofer, J. Eby, S. Gori, M. Lotito, M. Martone and D. Tuckler, Collider Signatures of Flavorful Higgs Bosons, Phys. Rev. D 94 (2016) 115032 [arXiv: 1610.02398] [INSPIRE]. 